\title{
THE POTENTIAL CONTRIBUTION OF FLOW CYTOMETRIC ANALYSIS IN DIAGNOSIS AND SCORING OF MYELODYSPLASTIC SYNDROME PATIENTS
}

\author{
Hesham M. Omar ${ }^{1}$; Amira R. El-Seikh ${ }^{1}$; Ayman Fathy ${ }^{2}$ and Amira. M. El-Helaly ${ }^{1}$ \\ ${ }^{1}$ Clinical Pathology and ${ }^{2}$ Intrernal medicine Department, Faculty of Medicine, Zagazig University
}

\begin{abstract}
Background: Myelodysplastic syndromes (MDSs) are a heterogeneous group of bone marrow disorders. Morphology may be difficult to evaluate in some patients, either due to hypocellularity or fibrosis of bone marrow.

Objective: To define immunophenotypic pattern in MDS patients, quantify and score complex flow cytometric abnormalities, and to correlate flow cytometry scores with conventional and established parameters used in MDS diagnosis.

Methods: Using flow cytometry, we studied 40 bone marrow specimens 30 patients with MDS and 10 cytopenic patients with non-clonal hematologic disorders as control group. A panel of monoclonal antibodies including: CD45, CD13, CD33, CD34, CD7, CD11b, CD16, CD56 and HLA-DR were used. Immunophenotyping using 2 gates were done: Forward (FSC) and right angle light scatter SSC and a second gate were done on the basis of CD45 staining and SSC to confirm identification of the selected cells. These combinations of Moab were used to measure the degree of maturation of their corresponding antigen by expressing their intensity.

Results: For MDS diagnosis, FCM had a sensitivity and specificity of $86.7 \%$ and $80 \%$ with score $1.5: 9,80 \%$ and $100 \%$ with score $3.5: 9,53.3 \%$ and $100 \%$ with score 5:9 and $30 \%$ and $100 \%$ with score $6.5: 9$ respectively, and a significant positive correlation between IPSS and FCM score.

Conclusion: This study defines immunophenotypic pattern in MDS patients and allow for a simple numerical display of results as score which is positively correlated with IPSS.
\end{abstract}

Key Words: Myelodysplastic syndromes; Flowcytometry score; Immunophenotypin

\section{INTRODUCTION}

$\mathbf{M}$ yelodysplastic syndrome (MDS) is a premalignant, clonal, haematopoietic stem cell disorder that features refractory cytopenia, ineffective hematopoiesis, dysplastic hematopoietic cell differentiation in multiple lineages of hematopoietic cells, and increased risk of progression to acute myeloid leukemia ${ }^{(\mathbf{1})}$. The diagnosis and management of patients with (MDSs) remain challenges that are distinct from those for most other hematologic malignancies. Therapeutic goals vary among individuals and are influenced by hematologic features, prognosis, and age. No single treatment is uniformly effective or addresses the clinical needs of all patients. The standard of care ranges from amelioration of hematologic deficit with blood product transfusions and administration of recombinant growth factors to aggressive chemotherapy and stem cell transplantation for younger individuals with more aggressive disease ${ }^{(2)}$.

The aim of this work is to define immunophenotypic patterns in MDS patients and quantify and score complex flow cytometric abnormalities, then correlate flow cytometry scores with conventional and established parameters used in MDS diagnosis.

\section{MATERIALS AND METHODS}

This study comprised 30 patients who were diagnosed as MDS selected from Clinical Pathology Department and Medical Oncology Unit, Internal Medicine Departments, Zagazig University Hospitals and The diagnosis of MDS was established based on WHO (World Health Organization) classification and derived from combined clinical, morphologic, and cytogenetic data., and ten bone marrow specimens were got from patients who admit to the hospital for ITP and/or hypersplenism diagnosis.

\section{Investigations:}

Full history taking and clinical examination: particularly for pallor, purpuric eruption, hepatomegaly, spleno-megaly and lymphadenopathy. Routine investigations were done for all studied subjects; Abdominal and pelvis ultra-sonography, Liver function tests, kidney function tests, uric acid and LDH using ADVIA 1650 (Bayer), Complete blood picture using Sysmex Kx 21(Roche) and examination of bone marrow aspirates (Leishman stain). Cytogenetic analysis were done for patient's group only. MDS patients were scored according to IPSS score. IPSS score is calculated based on marrow blast percentage, karyotype, and the number of cytopenias according to IPSS score.

Immunophenotyping was done using a panel of monoclonal antibodies labeled with FITC and PE including: CD45 ,CD13, CD33, CD34 CD7, CD11b , CD16, CD56 and HLADR using FACSCan flow cytometry (Becton Dickinson San Jose, CA). All mono-clonal antibodies used were purchased from Dako (Denmark), and the cut off for positivity for all was set at $20 \%$. These investigations were approved by the local Ethics Committees.

\section{METHODS}

IPSS score is calculated based on marrow blast percentage, karyotype, and the number of cytopenias according to IPSS score. 
IPSS Score.

\begin{tabular}{|c|c|c|c|c|c|}
\hline Prognostic Variable & $\mathbf{0}$ & 0.5 & 1 & 1.5 & 2 \\
\hline BM blasts \% & $<5$ & $5-10$ & & $11-20$ & $21-30$ \\
\hline $\begin{array}{l}\text { Karyo-type } \\
\text { Cyto-penias }\end{array}$ & $\begin{array}{c}\text { Good } \\
0 / 1\end{array}$ & $\begin{array}{c}\text { Interm-ediate } \\
2 / 3\end{array}$ & $\begin{array}{c}\text { Poor } \\
\ldots\end{array}$ & & \\
\hline
\end{tabular}

(Greenberg et al., 1997).

The total score is added, and the patient is staged according to the following:

- Low: 0

- Intermediate I : 0.5-1

- Intermediate II : 1.5-2

- High: Greater than or equal to 2.

Sampling:

$0.5 \mathrm{ml}$ of bone marrow aspirate was withdrawn under aseptic precautions delivered to EDTA vacutainer tube.

Flowcytometry procedures:

- $100 \mu \mathrm{L}$ of the anticoagulated blood containing up to $10^{4}$ cells were delivered to each of test tubes, $10 \mu \mathrm{L}$ of each monoclonal antibody

- To its specific tube were added and mixed gently, Incubated in the dark for 30 minutes. 1-2 ml erythrocyte lysing reagent were added to each tube and mixed gently, Centrifuged at $1200 \mathrm{rpm}$ for 5 minutes, then the supernatant was aspirated and $3 \mathrm{ml}$ $0.01 \mathrm{~mol} / \mathrm{L}$ PBS were added, vortexed gently,
Centrifuged at $1200 \mathrm{rpm}$ for 5 minutes, and then the supernatant was aspirated, leaving approximately $50 \mathrm{UL}$ of fluid.

- 10,000 to 15,000 total events were acquired from each tube forward and side scatter signals. Data were collected and processed using CellQuest software (Becton Dickinson, San Jose, CA).

- Two gates were done: Forward (FSC) and right angle light scatter SSC was done on monocytes and granulocytes as regard size and granularity.

- Second gate was done on the basis of CD45 staining and SSC to confirm identification of the selected cells and to measure the intensity of CD45 on the 2 groups of cells.

- The pattern of CD13 and CD16 (indicate maturational abnormalities) were measured as the degree of departure from normal antigen expression.

- Flow cytometric score was done for each case using the following score ${ }^{(4) .}$

\section{Flow Cytometric Score for MDS Diagnosis}

\begin{tabular}{cl}
\hline Points & Abnormality \\
\hline $\mathbf{0}$ & Appropriate CD45 SSC \\
& Normal relation of CD13 \&CD16 \\
& CD11 $^{+}$\&HLADR \\
& Negative lymphoid markers \\
& Single abnormality of the following in either myeloid or monocytic series : \\
& Abnormal granularity. \\
& CD 45 decreased intensity. \\
& HLADR+ve or CD11b -ve. \\
& CD56+ve. \\
& CD33-ve. \\
& CD13-CD16 convex pattern in granulocytes or loss of one on monocyte \\
& 2:3 of the above abnormalities or CD $34^{+}$or positive lymphoid marker on granulocytes or monocytes \\
& 4 or more of the above abnormalities or 1 or more plus CD34 ${ }^{+}$or positive lymphoid marker on \\
& granulocytes or monocytes \\
& Both myeloid cells and monocytes showed 2 or 3 abnormalities. \\
$\mathbf{4}$ &
\end{tabular}

One additional point was given if myeloblasts were less than $5 \%$ but clearly abnormal. Two additional points were given for $5 \%$ to $10 \%$ abnormal myeloblasts, 3 points for $11 \%$ to $20 \%$, and 4 points for $21 \%$ to $30 \%$ (there were no patients with more than $5 \%$ myeloblasts that had phenotypically normal myeloblasts). Total scores reflecting the degree of flow cytometric aberrancies were grouped into the following categories: $0-1$ normal/mild ; 2-3 moderate ; 4-9 sever.

Statistical analysis: 
Data analysis was done using the computer software statistical package for social sciences (SPSS) version 17.0.

Fisher exact (x 2) test was used for categorical variables. Pearson's correlation coefficient (r) was used to test the correlation between continuous variables.

P-value 0.05 was considered significant and 0.001 was considered highly significant.

\section{Results}

There was a significant statistical difference between the two groups as regards karyotyping $(\mathrm{P}<0.05)$ (table1). Karyotping for patient group reveals presence of 15 patients with normal karyotype, 5 patients with trisomy 8,3 patients with complex karyotype, 2 patients with monosomy 7,2 patients with monosomy 3, 2 patients with monosomy 20 and one patient with 5 q- syndrome . The number and percentage of patients with 5q-, RA, RAEB I and RAEB II were 1 (3.3\%), 12 (40\%), $7(23.3 \%)$ and $1033.3 \%$ respectively (table 2 ).
There was a significant positive correlations between IPSS score and karyotying $(\mathrm{r}=0.853, \mathrm{P}=$ $0.000), \quad \operatorname{LDH}(\mathrm{r}=0.690, \mathrm{P}=0.000)$ and $\mathrm{BM}$ blast ( $\mathrm{r}$ $=0.824, \quad \mathrm{P}=0.000)$ and significant negative correlations between IPSS score and TLC ( $\mathrm{r}=-0.376$, $\mathrm{P}=0.04)$, and PLT $(\mathrm{r}=-0.636, \mathrm{P}=0.000)$, No correlation between FCM score with the same parameters (table 3). Highly significant positive correlations between FCM score and IPSS score ( $\mathrm{r}$ $=0$. 0.582, $\mathrm{P}=0.001)$, presence of lymphatic markers on granulocytes $(\mathrm{r}=0.433, \mathrm{P}=0.017$ ), CD11b ( $\mathrm{r}=0.682, \mathrm{P}=0.000)$, presence of lymphatic markers on monocytes $(\mathrm{r}=0.585, \mathrm{P}=0.001)$,number of FCM abnormalities on granulocytes $(r=0.371, \mathrm{P}=$ 0.043), Number of FCM abnormalities on monocytes $(r=0.548, \mathrm{P}=0.002)$ (table 4). FCM score had sensitivity and specificity of $(86.7 \%, 80 \%)$ in score $1.5: 9,(80 \%, 100 \%)$ in score $3.5: 9,(53.3 \%$, $100 \%)$ in score 5: 9 and $(30 \%, 100 \%)$ in score 6.5: 9 (table 5).

Table (1): Comparison of laboratory data in the two studied groups as regards karyotyping.

\begin{tabular}{lccccccc}
\hline & \multicolumn{2}{c}{$\begin{array}{c}\text { Group I } \\
(\mathbf{n = 1 0})\end{array}$} & \multicolumn{2}{c}{$\begin{array}{c}\text { GroupII } \\
(\mathbf{n}=\mathbf{3 0})\end{array}$} & \multirow{2}{*}{$\boldsymbol{X}^{2}$} & P \\
\cline { 2 - 5 } & $\mathbf{N}$ & $(\boldsymbol{\%})$ & $\mathbf{N}$ & $(\boldsymbol{\%})$ & & \\
\hline Good & 10 & 100 & 15 & 50 & & \\
Intermediate & 0 & 0 & 7 & 23.3 & 8 & $0.018^{*}$ \\
Poor & 0 & 0 & 8 & 26.6 & & \\
\hline
\end{tabular}

*Significant

Table (2): Numbers and percentages of MDS subgroups

\begin{tabular}{|c|c|c|}
\hline Group II & Number & percentage \\
\hline $5 q-$ & 1 & $3.3 \%$ \\
\hline $\mathbf{R A}$ & 12 & $40 \%$ \\
\hline RAEB I & 7 & $23.3 \%$ \\
\hline RAEB II & 10 & $33.3 \%$ \\
\hline
\end{tabular}

Table (3): Correlation between IPSS \& FCM scores and other laboratory findings in Group II.

\begin{tabular}{lcccc}
\hline \multirow{2}{*}{$\begin{array}{c}\text { Group II } \\
(\mathbf{n}=\mathbf{3 0})\end{array}$} & \multicolumn{2}{c}{ IPSS Score } & \multicolumn{2}{c}{ FCM Score } \\
\cline { 2 - 5 } & $\mathbf{r}$ & $\mathbf{P}$ & $\mathbf{r}$ & $\mathbf{p}$ \\
\hline Karyotyping & 0.853 & $0.000^{*}$ & 0.16 & 0.935 \\
LDH & 0.690 & $0.000^{*}$ & -0.309 & 0.097 \\
TLC & -0.376 & $0.04^{*}$ & 0.189 & 0.318 \\
ANC & -0.214 & 0.255 & 0.084 & 0.661 \\
HB & -0.235 & 0.212 & -0.187 & 0.323 \\
PLT & -0.636 & $0.000^{*}$ & 0.213 & 0.257 \\
BM blast \% & 0.824 & $0.000^{*}$ & -0.113 & 0.550 \\
\hline
\end{tabular}

*Significant 
Table (4): Correlation between FCM\& IPSS scores and each item in FCM score in Group II.

\begin{tabular}{|c|c|c|c|c|}
\hline \multirow{2}{*}{$\begin{array}{c}\text { Group II } \\
(\mathbf{n}=\mathbf{3 0})\end{array}$} & \multicolumn{2}{|c|}{ FCM Score } & \multicolumn{2}{|c|}{ IPSS Score } \\
\hline & $\mathbf{R}$ & $\mathbf{P}$ & $\mathbf{r}$ & $\mathbf{P}$ \\
\hline IPSS & $0.582(* *)$ & 0.001 & & \\
\hline CD45 & 0.243 & 0.196 & 0.190 & 0.315 \\
\hline CD13/CD16 & 0.349 & 0.177 & 0.360 & 0.051 \\
\hline HLADR & -0.040 & 0.835 & -0.058 & 0.759 \\
\hline CD56 (Gran) & -0.035 & 0.854 & 0.172 & 0.364 \\
\hline CD34(Gran) & 0.288 & 0.123 & 0.005 & 0.978 \\
\hline Lymphatic markers(Gran) & $0.433(* *)$ & 0.017 & 0.089 & 0.639 \\
\hline Number of FCM abnormalities on granulocytes & $0.371(* *)$ & 0.043 & 0.295 & 0.114 \\
\hline CD56 (Mono) & 0.179 & 0.344 & 0.300 & 0.107 \\
\hline CD7 (Mono) & 0.019 & 0.922 & -0.124 & 0.513 \\
\hline CD34 (Mono) & 0.118 & 0.535 & -0.037 & 0.845 \\
\hline Lymph marker(Mono) & $0.585(* *)$ & 0.001 & 0.279 & 0.257 \\
\hline CD11b(Mono) & $0.682(* *)$ & 0.000 & -0.113 & 0.550 \\
\hline Number of FCM abnormalities on monocytes & $0.548(* *)$ & 0.002 & 0.16 & 0.935 \\
\hline
\end{tabular}

*Significant **highly significant

Table (5): Sensitivity and specificity for flowcytometric scores among MDS patients and control.using IPSS as gold standard test.

\begin{tabular}{lcccc}
\hline Cut point & sensitivity & specificity & $\begin{array}{c}\text { positive } \\
\text { predictive } \\
\text { value }\end{array}$ & $\begin{array}{c}\text { negative } \\
\text { predictive value }\end{array}$ \\
\hline $1.5: 9$ & $86.7 \%$ & $80 \%$ & $92.9 \%$ & $66.7 \%$ \\
$3.5: 9$ & $80 \%$ & $100 \%$ & $100 \%$ & $62.5 \%$ \\
$5: 9$ & $53.3 \%$ & $100 \%$ & $100 \%$ & $41.7 \%$ \\
$6.5: 9$ & $30 \%$ & $100 \%$ & $100 \%$ & $32.3 \%$ \\
\hline
\end{tabular}

\section{DISCUSSION}

Myelodysplastic syndromes $\left(\mathrm{MDS}_{\mathrm{s}}\right)$ are premalignant, clonal, haematopoietic stem cell disorder that features refractory cytopenia, ineffective hematopoiesis, dysplastic hematopoietic cell different-iation in multiple lineages of hematopoietic cells, and increased risk of prog-ression to acute myeloid leukaemia. ${ }^{(\mathbf{1})}$.

The diagnosis and management of patients with $\left(\mathrm{MDS}_{\mathrm{s}}\right)$ remain challenges that are distinct from those for mostother hematologic malignancies therapeutic goals vary among individuals and are influenced by hematologic features, prognosis, and age.No single treatment is uniformly effective or addresses the clinical needs of all patients ${ }^{(2)}$.

The objective of the present study was to define immunophenotypic pattern in MDS patients and quantify and score complex flow cytometric abnormalities, then correlate flow cytometry scores with conventional and established parameters used in MDS diagnosis.

This study was carried out on 30 patients with a diagnosis of MDS entering oncology department (confirmed at our laboratory), and 10 healthy subjects as a control.

MDS subgroups in this study were divided into 5qwas (3.3\%) ,RA was (40\%), RAEB I (23.3\%) and RAEB II was ( $33.3 \%)$,meanwhile Xu et al., (2013) $^{(3)}$ found $\operatorname{RA}(14.2 \%), \operatorname{RCM}(42 \%), \mathrm{RCEBI}$ (28.2\%),RAEBII(14\%),MDS-U(1.7\%),5q- (0\%).

A significant positive correlation in group II between IPSS score and karyotying ( $\mathrm{P}=0.000)$, LDH ( $\mathrm{P}=0.000)$ and BM blast $(\mathrm{P}=0.000)$ and significant negative correlation between IPSS score and TLC( $\mathrm{P}=0.04)$, and PLT ( $\mathrm{P}=0.000)$ as these parameters were included in IPSS score .No correlation between IPSS and ANC, Hb, CD45, CD13/CD16, CD56, HLADR, CD34,presence of lymphatic marker on granulocyte and on monocytes, CD7, CD11b

In MDS group we found significant positive correlation between FCM score and IPSS score ( $\mathrm{P}=$ 0.001), Presence of lymphatic markers on granulocytes( $\mathrm{P}=0.017) \quad, \mathrm{CD} 11 \mathrm{~b}(\mathrm{P}=0.000)$, Presence of lymphatic markers on monocytes $(\mathrm{P}=$ 0.001),number of FCM abnormalities on 
granulocytes( $\mathrm{P}=0.043)$, number of FCM abnormalities on monocytes $(\mathrm{P}=0.002)$. These results coincide with the results reported by $\mathrm{Xu}$ et al.(,2013) $)^{(\mathbf{3})}$ who reported a positive correlation between FPS score and IPSS (Spearman $r=0.498$, $\mathrm{P}<0.001$ ).

We found no correlation between FCM score and karyotypic abnormalities, BM blasts and peripheral cytopenias .Wells et al., (2003) $)^{(4)}$ disagree with our results and find significant increase in flow cytometry scores with increasing cytogenetic risk by IPSS criteria $(\mathrm{P}=0.02)$.However, for a number of patients, flow scores and IPSS cytogenetic categories were disparate where $42 \%$ of patients with normal cytogenetics (good risk) had flow scores of 4 or higher. Xu et al., ( 2014) ${ }^{(5)}$ reported that FCM score and advanced scoring showed significant correlation with cytogenetic prognosis (Spearman $\mathrm{r}=0.379, \mathrm{P}, 0.001$ ), but early scoring had an inverse correlation with cytogenetic prognosis (Spearmanr $=20.213, \mathrm{P}=0.004$ ) Therefore, FCM scores divided into early scoring and advanced scoring could contribute to disease classification, stage evaluation and treatment options for MDS.

So this disagreement with our results may be explained with smaller number of cases in our study and may be due to we did not divide the FCM score to early and advanced score.

Xu et al.,(2013) ${ }^{(3)}$ agree with our results, found no correlation between FCM score and peripheral cytopenias(-0.076, $\mathrm{P}=0.455)$.

Our FCM score had sensitivity and specificity of ( $86.7 \%, 80 \%)$ in score $1.5: 9,(80 \%, 100 \%)$ in score $3.5: 9,(53.3 \%, 100 \%)$ in score $5: 9$ and $(30 \%, 100 \%)$ in score 6.5: 9. This result was close to the results of Della Porta (2012) ${ }^{(\mathbf{6})}$ who reported a FCMSS with $69 \%$ of sensitivity and $92 \%$ of specificity, and Xu et al .,(2014) ${ }^{(5)}$ also agree with our results with sensitivity of $89.4 \%$ and specificity of $96.5 \%$ for score 3. Conversely other studies show different results, Wells et al $\mathbf{( 2 0 0 3 )})^{(\mathbf{4})}$ reported a FCMSS with $54.8 \%$ of sensitivity and $100 \%$ of specificity in diagnosing MDS. Ogata et al (2006) ${ }^{(7)}$ designed a FCMSS to diagnose low-grade MDS based on immunophenotyping in CD34+ cells, which showed $58 \%$ of sensitivity and $100 \%$ of specificity.

The inter-laboratory bias may have negative effect on the diagnostic power. It is still difficult to construct standardized FCM detection assay in different labs. The

\section{RECOMMENDATIONS}

- Further studies should be done to refine the FCSS by incorporating additional antibodies; the methodology must remain practical if it is to be applied routinely.
- efforts should be directed at expanding our abilities to phenotypically chara-cterize the erythroid compartment

- More studies on large numbers of patients are needed to confirm these results.

\section{CONCLUSION}

Our scoring system tested the components of myeloid maturation separately monocytes, and neutrophils

- The phenotypic abnormalities on the maturing myeloid and monocytoid are abnormalities detected in MDS patients were different from individual to individual prompting a scoring system based on the sum of the abnormalities rather than focusing on individual antigenic differences.

biological signals of abnormal maturation. Based on the abnormal pattern, we developed and validated a convenient and economical FCM score diagnostic system with good sensitivity and specificity to assist the diagnosis of MDS

\section{Acknowledgments:}

We wish to thank the members of Clinical Pathology Department, Faculty of Medicine, Zagazig University and every one helped us to complete this work. Without their continued efforts and support, we would have not been able to bring our work to a successful completion.

\section{REFERENCES}

1. Samuel J, Pirrucello et al. Myloblast phenotypic changes in myelody-splasia: CD34 and CD117 expression abnormalities are common. Am .J. Clin. Path (2006), 125: 884-894.

2. List F,Sanberg A. and Donald C. Doll : Myelodysplastic syndromes in:Wintrobe $\mathrm{s}$ clinical hematology «12th ed. »:John P.Greer,Johon Foerster,George M.Rodgers eds. (2009) vol.5, $1956: 1987$.

3. Feng $\mathrm{Xu}$, Juan Guo, Ling-Yun $\mathrm{Wu}$ et al., Diagnostic Application and Clinical Significant of FCM Progress Scoring System Based on Immuno-phenotyping in CD341Blasts in Myelodysplastic Syndromes Cyto-metry Part B (Clinical Cytometry) (2013) 84B:267-278 .

4. Wells DA, Benesch M, Loken MR, et al. Myeloid and monocytic dyspoiesis as determined by flow cytometric scoring in myelody-splastic syndrome correlates with the IPSS and with outcome after hematopoietic stem cell transplantation. Blood (2003); 102: 394-403.

5. Xu F, Li X, Chang C-K, Guo J, Wu L-Y, et al. Establishment and Validation of an Updated Diagnostic FCM Scoring System Based on Pooled Immunophenotyping in CD34+Blasts and Its Clinical Significance for Myelodysplastic Syndromes. PLoS ONE (2014) 9 (2): e88706. 
6. Della Porta MG, Picone C, Pascutto C, Malcovati L, Tamura $\mathrm{H}$, et al. Multicenter validation of a reproducible flow cytometric score for the diagnosisof low-grade myelodysplastic syndromes: results of a European Leukemia NET study. Haematologica (2012) 97: 1209-1217.

7. Ogata K, Kishikawa Y, Satoh C, Tamura H, Dan $\mathrm{K}$, et al. Diagnostic application of flow cytometric characteristics of CD34+ cells in lowgrade myelodysplastic-syndromes. Blood (2006) 108: 1037-1044.

8- Greenberg P,Cox C,Le Beau MM ,Fenaux P,Morel P,Sanz G, al.International scoring system for evaluating prognosis in myelodysplastic Syndromes Blood (1997);89:2079-2088. 